presented with leg weakness and pain, followed in 2 to 6 days by loss of pain and temperature sensation, and finally complete myelopathy, with proprioceptive loss and urinary incontinence. One patient had brain involvement also, manifesting with delirium, spasticity, and arm weakness. Despite aggressive antifungal therapy, the patients died within 1 month of onset of symptoms. Postmortem findings showed multifocal meningeal exudates, cerebral and spinal hemorrhagic necrosis, and fungal abscess in the cord of one child, and aortic endarteritis, spinal necrosis and intramedullary hematoma in a second. A third, without postmortem, had shown CT and MRI evidence of a paravertebral mass with vertebral osteomyelitis, and a positive biopsy culture for Aspergillus. Blood and CSF cultures were negative. (Koh S, Ross LA, Gilles FH, Nelson MD Jr, Mitchell WG. Myelopathy resulting from invasive aspergillosis. Pediatr Neurol Aug 1998;19:135138). (Respond: Dr Susan Koh, Miami Children's Hospital, Neuroscience Center, 3100 SW 62nd Avenue, Miami, FL 33155).

COMMENT. Aspergillosis is a potential cause of myelopathy in immunosuppressed children treated with broad spectrum antibiotics.

\title{
CEREBRAL CANDIDIASIS SEQUELAE IN PREMATURE INFANTS
}

Cerebral candidiasis in two premature infants, severely handicapped on follow-up at 3 and 6 years, is reported from the Women's College Hospital, Toronto, Canada. Infant 1 had respiratory distress syndrome and was treated with ampicillin and gentamicin for 5 days. A Candida diaper rash with skin breakdown on day 12 was complicated by sepsis, treated with vancomycin and cefotaxime, and followed by systemic candidiasis, requiring amphotericin and 5-flucytosine. Head ultrasound showed echogenic fungal microabscesses in brain parenchyma that resolved after 37 days treatment. Follow-up at 6 years revealed mild cerebral palsy, severe aphasia, and ADHD. Infant 2 had respiratory distress syndrome, low Apgars, skin rash, sepsis treated with ampicillin, vancomycin and gentamicin, and at 6 days, candidiasis treated with amphotericin and flucytosine. Head ultrasound showed scattered echogenic microabscesses, and later, calcifications in the basal ganglia and periventricular areas. At 3 year follow-up, the child had spastic quadriplegia, speech impairment, and cognitive delay. (Scott PA, Ohlsson A. Sequelae associated with cerebral candidiasis in two premature infants. Acta PaediatrOct 1998;87:1090-2). (Respond: Dr PA Scott, NICU, Women's College Hospital, 76 Grenville Street, Toronto, Ontario, Canada M5S 1B2).

COMMENT. Previous reports of candidiasis involving the brain have involved autopsy descriptions of scattered microabscesses. Large doses of amphotericin $(1 \mathrm{mg} / \mathrm{kg} / \mathrm{d})$ and 5-flucytosine were thought to explain the survival of the two infants described above, although the resolution of infection did not prevent significant neurologic handicaps as sequelae. Treatment was continued for 37 days in case 1, with the better outcome, and 22 days in case 2, more severely handicapped. Perhaps more prolonged treatment may have improved the outcome, but serious side effects can be a problem. AAP 1997 Redbook recommends 6 weeks or longer treatment for systemic candidiasis in high risk patients.

\section{TOXOPLASMIC ENCEPHALITIS AND HYPER-IgM SYNDROME}

A 9-year-old boy with hyper-IgM syndrome complicated by impaired consciousness and disseminated toxoplasmosis is reported from Nagoya University School of Medicine, Japan. X-linked hyper-IgM syndrome was diagnosed at 5 months of age and treated with IV immunoglobulin. On admission, he had involuntary movements and convulsions. CT and MRI showed multiple lucencies 